\title{
Problemas, situaciones de riesgo y vivencias traumáticas de adolescentes escolarizados
}

\author{
Problems, risk situations and traumatic experiences of school teenagers
}

\author{
Carmen Marina Arrom Suhurt $\mathbb{D}^{1}$, Pamela Ortega $\mathbb{Q}^{2}$, María Auxiliadora Arrom Suhurt $\mathbb{Q}^{1}$, María del Pilar Fresco $\mathbb{Q}^{3}$, \\ Mónica Ruoti $\mathbb{D}^{1}$, Margarita Samudio $\mathbb{D}^{4}$, Cristina Arrom Suhurt $\mathbb{B}^{4}$
}

${ }^{1}$ Centro para el Desarrollo de la Investigación Científica, Asunción, Paraguay.

¿Univerisdad Nacional de Asunción, Facultad de Ciencias Médicas, San Lorenzo, Paraguay.

3Universidad Católica Nuestra Señora de la Asunción, Asunción, Paraguay

${ }^{4}$ Instituto de Investigaciones en Ciencias de la Salud, San Lorenzo, Paraguay

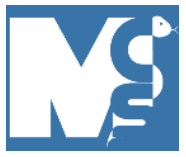

Recibido: $11 / 06 / 2020$

Revisado: $18 / 08 / 2020$

Aceptado: 04/12/2020

\section{Autor correspondiente}

Carmen Marina Arrom Suhurt Centro para el Desarrollo de la Investigación Científica marinaarrom@yahoo.com.ar

\section{Conflictos de interés}

Los autores declaran no poseer conflictos de interés.

\section{Fuente de financiación}

Los autores no recibieron apoyo financiero para la investigación, autoría y/o publicación de este artículo.

Este artículo es publicado bajo una licencia de Creative Commons Reconocimiento 4.0 Internacional.

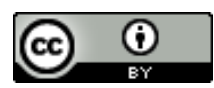

\section{RESUMEN}

Introducción: La OMS señala que en el 2016 murieron en el mundo aproximadamente 1,1 millones de adolescentes de entre 10 y 19 años, en su mayoría, por causas prevenibles. Objetivo: El presente estudio buscó analizar las problemáticas, situaciones de riesgo y vivencias traumáticas que experimentaron los adolescentes estudiantes de escolar básica y bachillerato durante el último año. Metodología: El diseño fue no experimental, cuantitativo descriptivo de corte retrospectivo. Participaron 661 adolescentes de 56 colegios de Alto Paraná a través de un muestreo probabilístico estratificado por conglomerado trietápico. Con previa autorización de los padres, se utilizó el cuestionario auto-administrado Plan de Acción de Desarrollo y Salud de Adolescentes y Jóvenes en las Américas de la Organización Panamericana de la Salud adaptado a los fines de la investigación. Resultados: Los resultados reflejaron situaciones problema en el hogar como discusiones con su madre/padre (44,6\%), mal comportamiento y desobediencia (35,1 \%). A nivel académico, notas bajas en colegio (43,1\%). De forma transversal, problemas de caráctertemperamento $(25,4 \%$ y consumo de alcohol $(21,0 \%)$. En cuanto a las vivencias emocionales, $33,1 \%$ se sintió triste o nervioso(a) todo el tiempo; $17,4 \%$ se involucró en peleas o riñas. Conclusión: Se evidenciaron situaciones de riesgo como intento de suicido (16,6 \%); y vivencias traumáticas como abuso sexual $(6,5 \%)$ golpes y maltrato (16,0\%). Solo un $10,9 \%$ acudió a consulta por problemas emocionales.

Palabras clave: estudiantes; adolescentes; autopercepción; riesgo

\section{ABSTRACT}

Introduction: The WHO indicates that in 2016 approximately 1.1 million adolescents between the ages of 10 and 19 died in the world, mostly due to preventable causes. Objective: The present study analyzed the problems, risk situations and traumatic experiences experienced by teens in elementary school and high school during the last year. Methodology: The design was non-experimental, quantitative, descriptive, retrospective. 661 adolescents from 56 schools in Alto Paraná participated through a probabilistic sampling stratified by three-stage conglomerate. With the prior authorization of the parents, the self-administered questionnaire Plan of Action for Development and Health of Adolescents and Young People in the Americas of the Pan American Health Organization adapted for the purposes of the research was used. Results: The results reflected problematic situations at home such as arguments with their mother / father (44.6\%), bad behavior and disobedience (35.1\%). At an academic level, low grades in school (43.1\%). In a transversal way, character-temperament problems $(25.4 \%)$ and alcohol consumption $(21.0 \%)$. Regarding the emotional experiences, $33.1 \%$ felt sad or anger all the time; $17.4 \%$ got involved in fights. Conclusion: Risk situations such as suicide attempt (16.6\%) were evidenced; and traumatic experiences such as sexual abuse (6.5\%), beatings and mistreatment (16.0\%). Only $10.9 \%$ attended the consultation for emotional problems.

Keywords: students; teenagers; self-perception; risk. 


\section{INTRODUCCIÓN}

Una sexta parte de la población en el mundo está constituida por adolescentes y es sabido que en general conviven en buen estado de salud, aunque la mortalidad prematura, las enfermedades y lesiones constituyen hasta hoy un problema relevante, porque estas últimas socaban las posibilidades de su desarrollo integral (1). Entre las lesiones que las afectan, aquellas producidas en accidentes de tránsito representan la más importante causa de muerte en adolescentes, aparte del suicidio, la violencia, el VIH Sida y otras enfermedades como las diarreicas y trastornos de salud mental que no se identifican ni tratan a tiempo $(1,2)$.

En el mismo marco de la problemática, es importante señalar el estudio de Mayorga realizado en Madrid, España, con 466 adolescentes escolarizados de ambos sexos, señalando la evidencia que quienes percibían conflictos entre los padres, eran más proclives a presentar problemas de conducta que los que no (3). Los autores remarcaron el hallazgo de una asociación entre conflictos parentales y problemas independientemente de la estructura familiar (3).

También en España un estudio en 1510 adolescentes de ambos sexos escolarizados de nueve centros de enseñanza, mostró como problemática, agresión hacia los iguales, sintomatología depresiva, estrés percibido y soledad y bajo nivel de autoestima, satisfacción con la vida y empatía (4).

Tomando en consideración el panorama mundial y los estudios en España sobre los problemas de salud integral de adolescentes, la perspectiva de la problemática en América Latina se puede visualizar en otros estudios. Al respecto, en un trabajo realizado en Manizales-Colombia con población adolescente escolarizada (5) se encontró un factor de riesgo suicida de $16,5 \%$, y como factores asociados, acoso o intimidación escolar (bullying), disfuncionalidad familiar, baja autoestima, depresión, trastornos de la conducta alimentaria, consumo de cigarrillo, dependencia alcohólica y consumo de sustancias psicoactivas (5). En la misma línea, otra investigación realizada en Santa Marta, Colombia, en estudiantes de secundaria con bajo rendimiento escolar mostró que los mismos presentaban factores psicológicos negativos que los afectaban en su rendimiento y aprendizaje escolar, pero principalmente su salud mental y bienestar psicológico (6).

En el mismo orden de ideas, datos relevantes de una investigación llevada a cabo por Fernández en la Plata, Argentina con 120 pacientes adolescentes de ambos sexos en un Hospital Especializado en
Drogodependencia y Alcoholismo, encontró que el 63\% se inició en el consumo de sustancias entre los 12 y 15 años (7). Y sigue citando el autor el hallazgo de otras conductas de riesgo que se encontraron en contexto de abandono, indiferencia, marginalidad, ausencia de una figura de autoridad en el vínculo entre padres e hijos (7).

Respecto a la problemática del consumo adolescente de drogas en Paraguay, la Dirección de Prevención Integral y la Dirección de Desarrollo Alternativo aplicó encuestas sobre esta problemática en adolescentes del interior del país (8). Los resultados parciales correspondientes al Departamento de Alto Paraná revelaron que el inicio del consumo de cigarrillos y alcohol en estudiantes se da a partir de los 13 años. Un alto número de jóvenes se inicia con las drogas lícitas siendo aún menores de edad y en etapa en que deberían estar bajo el cuidado de sus padres o tutores (8).

El presente estudio se propuso analizar las problemáticas, situaciones de riesgo y vivencias traumáticas que experimentaron adolescentes de escolar básica y educación media durante el último año.

\section{METOdOLOGÍA}

Estudio descriptivo, retrospectivo (9) de adolescentes de 56 colegios de Alto Paraná. El muestreo fue probabilístico y trietápico, cuyo desarrollo fue el siguiente: En la primera etapa fueron seleccionados los colegios en forma sistemática, con arranque aleatorio y probabilidad proporcional al tamaño de cada colegio. En la segunda etapa fueron electos los cursos dentro de cada colegio en forma sistemática con arranque aleatorio y probabilidad aproximada al tamaño de cada curso. En la tercera etapa, fueron seleccionados los alumnos con igual probabilidad de inclusión, en base a la lista proporcionada por el Ministerio de Educación y la Supervisión de cada ciudad.

Respecto al cálculo del tamaño muestral se utilizó el paquete Epidat (Organización Panamericana de la Salud, Junta de Salud de Galicia y Universidad CES de Colombia) para establecer la proporción esperada de adolescentes con distintas problemáticas en 0,50 , con un error de muestreo $p=0,04$ y un nivel de confianza $95 \%$, el tamaño de la muestra es de 600 , a lo que se agregó un excedente de tomas, en previsión de invalidaciones y reemplazos. La muestra fue cerrada en 661 casos para su procesamiento estadístico (9).

Se completó un cuestionario auto-administrado, estructurado, anónimo previa autorización de los 
padres. Se solicitó a estudiantes a participaren de forma voluntaria, proporcionándoles información necesaria sobre la administración de los resultados y los objetivos de la investigación. Como instrumento se utilizó un cuestionario adaptado del Plan de Acción de Desarrollo y Salud de Adolescentes y Jóvenes en las Américas de la Organización Panamericana de la Salud (10) con variables de problemáticas, situaciones de riesgo y traumáticas que pudieran haber vivido en el transcurso del año anterior. Se incluyeron además variables socio demográficas (sexo, grupo etario, escolaridad, tipo de colegio, actividad laboral remunerativa, personas con quienes vive).

Control de calidad de los datos: como método de control de calidad, el instrumento de campo fue sometido a prueba piloto con una muestra de 50 estudiantes de Alto Paraná que fueron excluidos posteriormente en la aplicación definitiva. Posterior a este paso, se elaboró la versión final. Se realizó la crítica a cada cuestionario a fin de detectar inconsistencias. Posteriormente fueron preparados para su digitalización.

Consideraciones Éticas: en los meses previos a la implementación, se obtuvo una autorización del Ministerio de Educación y Ciencias (MEC) que declaró de interés el desarrollo del estudio. Posteriormente, desde cada Institución (56 colegios) se envió una Nota a padres y madres para solicitar su autorización. La participación de los/las adolescentes fue voluntaria, proporcionándoles información suficiente y clara sobre la utilización de los datos y fines de la investigación. En todos los casos se guardó escrupulosamente la confiabilidad de las informaciones obtenidas.

Los autores declaran que no tienen conflictos de interés comercial. El presente estudio no cuenta con financiación, independientemente del Incentivo a Investigadores PRONII.

\section{RESULTADOS}

Del total de la población de estudiantes (661), 343 $(51,9 \%)$ se identificó con el sexo femenino, $318(48,1$ $\%)$ con el masculino; 281 (42,5\%) perteneció al grupo de edad de 12 a 15 años, 254 (38,4 \%) de 16 a 17 años y 122 (18,5\%) 18 a 19 años; se encontraban cursando la Escolar Básica 346 (52,4 \%) estudiantes y el Bachillerato 308 (46,5\%); pertenecían a Colegios Públicos 428 (64,8 \%) y a Colegios Privados 187 (28,2 $\%)$; se hallaban trabajando 147 estudiantes (22,3\%) (Tabla 1).

Al momento del estudio vivían con los padres 493 (74,6 $\%)$ estudiantes, con la madre $86(13,0 \%)$, con parientes $38(5,7 \%)$, con el padre $18(2,7 \%)$ y con el encargado no pariente $16(2,4 \%)$, el resto corresponde a amigos o compañeros.

TABLA 1. DISTRIBUCIÓN DE LA MUESTRA POR CARACTERÍSTICAS SOCIODEMOGRÁFICAS (N=661)

\begin{tabular}{lcc}
\hline \multicolumn{1}{c}{ Sexo } & Frecuencia & Porcentaje \\
\hline Masculino & 318 & 48,1 \\
Femenino & 343 & 51,9 \\
Grupo de edad & & \\
12 a 15 años & 281 & 42,5 \\
16 a 17 años & 254 & 38,4 \\
18 a 19 años & 122 & 18,5 \\
No responde & 4 & 0,6 \\
Escolaridad & $\mathrm{n}$ & $\%$ \\
Escolar Básica & 346 & 52,4 \\
Bachillerato & 308 & 46,5 \\
No contesta & 7 & 1,1 \\
Tipo de Colegio & & \\
Colegio Público & 428 & 64,8 \\
Colegio Privado & 187 & 28,2 \\
No responde & 46 & 7,0 \\
Trabaja actualmente & & \\
Sí & 147 & 22,3 \\
No & 514 & 77,7 \\
\hline
\end{tabular}

Entre los problemas o dificultades sobresalen discusiones con mamá/papá 44,6\% (295), notas bajas en el colegio $43,1 \%$ (285), mal comportamiento $y$ desobediencia en casa $35,1 \%$ (232), problemas de carácter-temperamento $25,4 \%$ (168) y consumo de alcohol 21,0\% (139). De las situaciones vividas personalmente o por algún miembro de su familia, el
$33,1 \%$ (219) se sintió triste o nervioso(a) todo el tiempo, $17,4 \%(115)$ se involucró en peleas o riñas alguna vez, 16,6\% (110) intentó quitarse la vida alguna vez, $16,0 \%$ (106) recibió golpes o maltrato; $10,9 \%$ (72) consultó por problemas emocionales alguna vez y $6,5 \%$ (43) fue víctima de abuso sexual. 


\section{DISCUSIÓN}

Cerca de la mitad de los/as adolescentes escolarizados del estudio manifestó como principales problemas o dificultades en el último año, haber tenido discusiones con su padre/madre y notas bajas en el colegio, a lo que le siguió en importante porcentaje, mal comportamiento y no obediencia a padres. Problemas de carácter y temperamento y consumo de alcohol tuvo un cuarto del grupo estudiado. La evidencia encontrada es coincidente con los resultados de la investigación de Rueda en Bucaramanga-Colombia, donde uno de cada siete estudiantes presentó problemáticas de consumo abusivo de alcohol y como factores asociados la influencia de amigos, la familia, la edad y el comportamiento antisocial $(11,12)$.

Los resultados del presente estudio también mostraron que un tercio del grupo padeció tristeza y nervios en forma permanente, y casi un quinto del grupo se involucró en peleas y riñas, intentó quitarse la vida alguna vez y recibió golpes y maltrato. Sólo una décima parte consultó por problemas emocionales alguna vez. También autores como Chávez y Hernández en 2017 señalaron situaciones similares en una investigación realizada en México (13) con problemas familiares (sentirse enojados, tristes, solos), deprimidos e incomprendidos. Resultados afines mostraron el estudio del Quindio-Colombia, donde un tercio de los estudiantes escolarizados afirmó padecer tristeza todo el día durante el último mes y exteriorizaron riesgo suicida moderado y alto (14).

Otros elementos encontrados fueron ideas de que su futuro es vago/incierto, problemas para satisfacer necesidades, desconfianza ante el apoyo de otros, y desesperanza ante cosas que ansían (14). En Ecuador, Moreta señaló en su estudio con adolescentes escolarizados riesgo elevado en la dimensión escolar, aspectos asociados a conductas problemáticas como repetición del año, dificultades en el aprendizaje o indiferencia y en la dimensión familia, problemas emocionales, conflictos con el sistema parental y desaprobación social (15).

En relación a la problemática del abuso sexual y el hallazgo de 43 casos en el presente estudio, se encontraron evidencias similares en la investigación de Àlape Zuluaga (16) realizada en una institución educativa de Manizales, Colombia. Resaltó en ésta el abuso sexual frecuente en población escolar (1 de cada 3 en niños, 1 de cada 5 en niñas) en edades comprendidas entre 12 y 14 años (16).

Respecto a la relación con el agresor se aprecia que la pequeña proporción de abuso intrafamiliar se localiza en el de alta sintomatología. Aunque la mayoría son abusados por integrantes de su familia, estos resultados sugieren que el abuso intrafamiliar estaría asociado a menor sintomatología de estrés postraumático. La mayor proporción de víctimas de abuso crónico se agrupó en el conglomerado de baja sintomatología. Los adolescentes de los 3 grupos no se diferenciaron mayormente en edad, tipo de abuso sexual sufrido, lapso de tiempo transcurrido desde el último episodio, latencia de la revelación, violencia utilizada por el agresor y existencia de otros sucesos traumáticos en los últimos 2 años (16).

El trabajo investigativo en adolescentes escolarizados de la ciudad de Necochea, Prov. de Buenos Aires, realizado por Di Rico, puso también en evidencia relaciones existentes entre riesgo suicida y factores interpersonales de soledad, aislamiento social y apoyo social percibido. El análisis de las correlaciones entre riesgo suicida y las dimensiones interpersonales reveló asociaciones significativas prácticamente en todos los casos, las de mayor magnitud con soledad y apoyo social percibido (17). La variable soledad, es decir la vivencia de insatisfacción -real o percibida- en las relaciones interpersonales, presentó las correlaciones positivas de mayor magnitud con los factores de riesgo baja autoestima, desesperanza, soledad y aislamiento e ideaciones suicidas, y también con riesgo suicida (17).

En cuanto al apoyo social percibido, se observan las asociaciones más elevadas con la presencia de ideaciones suicidas, el aislamiento social y el potencial de riesgo de suicidio. Los resultados indicaron que el apoyo social de los padres ejercería como un factor protector directo, y el que pareciera ejercer mayor efecto alude al acompañamiento parental activo en la vida del adolescente y ser fuente donde los jóvenes pueden buscar consejo y apoyo de sus elecciones (17).

Las causas que aluden los adolescentes para realizar tentativas suicidas son el inicio del desapego familiar y el ingreso al contexto colectivo. Los problemas familiares cumplen un papel fundamental en el riesgo suicida (13) y deben ser tomados en cuenta en instituciones educativas que pueden tener una mirada importante hacia las situaciones problemáticas padecidas por los y las jóvenes, detectadas o identificados en el contexto socioeducativo.

Como limitación del estudio se puede mencionar el hecho de ser retrospectivo, por lo que, la información respecto a las problemáticas visualizadas por adolescentes y las experiencias vividas pueden no corresponderse con la situación de hoy día. Políticas públicas enmarcadas en la atención integral a la adolescencia desde el Ministerio de la Niñez y la Adolescencia, así como desde el Ministerio de 
educación pueden ayudar a tener esta mirada del contexto micro y macro social de adolescentes. Políticas que brinden oportunidades de desarrollo y una vida digna a la población en general y a jóvenes en particular, con sus distintos contextos familiares.

\section{REFERENCIAS}

1. Organización Mundial de la Salud. Adolescentes: riesgos para la salud y soluciones [Internet]. 2018 [citado 23 de enero de 2021]. Disponible en: https://www.who.int/es/news-room/fact-

sheets/detail/adolescents-health-risks-and-solutions

2. Organización Mundial de la Salud. Suicidio [Internet]. 2019 [citado 23 de enero de 2021]. Disponible en: https://www.who.int/es/news-room/factsheets/detail/suicide

3. Mayorga Muñoz CJ, Godoy Bello MP, Riquelme Sandoval SV, Ketterer Romero LM, Gálvez Nieto JL. Relación entre Problemas de Conducta en Adolescentes y Conflicto Interparental en Familias Intactas y Monoparentales. Rev Colomb Psicol. 2016;25(1):107-22. Https://www.doi.org/10.15446/rcp.v25n1.48705

4. Estévez López E, Jiménez Gutiérrez TI. Conducta agresiva y ajuste personal y escolar en una muestra de estudiantes adolescentes españoles. Univ Psychol. 2014;14(1):111-24. Https://www.doi.org/10.11144/Javeriana.upsy14-1.caap

5. Castaño Castrillón JJ, Aguirre-Flórez DC, Cañón SC, MarínSánchez DF, Rodríguez-Pabón JT, Rosero-Pantoja LÁ, et al. Riesgo suicida y factores asociados en adolescentes de tres colegios de la ciudad de Manizales (Colombia), 2013. Rev Fac Med. 2015;63(3):419-29. Https://www.doi.org/10.15446/revfacmed.v63n3.44205

6. Ferrei Ortega FR, Vélez Mendoza J, Ferrel Ballestas LF. Factores psicológicos en adolescentes escolarizados con bajo rendimiento académico: depresión y autoestima. Encuentros. 2014;12(2):35-47. URL.

7. Fernández Raone Napolitano M. Adolescencia y consumo de sustancias: la demanda terapéutica en un centro especializado. Perspect En Psicol. 2016;13(2):84-94. URL.

8. ABC Color. Jóvenes se inician en drogas desde los 13 años [Internet]. 2015 [citado 23 de enero de 2021]. Disponible en: https://www.abc.com.py/nacionales/jovenes-iniciancon-drogas-desde-los-13-anos-1387956.html

9. Hernández Sampieri R, Fernández Collado C, Baptista Lucio $P$, Méndez Valencia S, Mendoza Torres CP. Metodología de la investigación. Mexico, D.F.: McGrawHill; 2014.

10. Organización Panamericana de la Salud. Estrategia y Plan de Accion Regional Sobre los Adolescentes y Jóvenes. OPS; 2010.

11. Rueda-Jaimes GE, Pinto Ramírez JL, Rangel MartínezVillalba AM, Camacho López PA. Alcohol abuse and associated factors in student children and adolescents. Rev Colomb Psiquiatr. 2012;41(2):273-83. URL.

12. Faria NMX, Victora CG, Meneghel SN, Carvalho LA de, Falk JW. Suicide rates in the State of Rio Grande do Sul, Brazil: association with socioeconomic, cultural, and agricultural factors. Cad Saúde Pública. 2006;22(12):2611-21. Https://www.doi.org/10.1590/S0102311X2006001200011

13. Chávez-Hernández AM, Correa-Romero FE, KleinCaballero AL, Macías-García LF, Cardoso-Espindola KV, Acosta-Rojas IB. Sintomatología depresiva, ideación suicida y autoconcepto en una muestra de niños mexicanos. Av En Psicol Latinoam. 2017;35(3):501-14. URL.

14. Salazar JAA, Pretel LD, Maya PAO, Avilés ER, Valencia JEC.
Autoestima y desesperanza en adolescentes de una institución educativa del Quindío. Duazary Rev Int Cienc Salud. 2017;14(2):179-87. URL.

15. Moreta R, Reyes C, Mayorga M, León-Tamayo L. Estimación sobre niveles y factores de riesgo psicosocial en adolescentes escolarizados de Ambato, Ecuador. Pensando Psicol. 2017;13(22):29-40.

Https://www.doi.org/10.16925/pe.v13i22.1986

16. Zuluaga $C$ julieth À, Castrillón J jaime castaño, Quintero L maría castaño, Enciso E francisco cely, Enao J marín, Arcía D gildardo ocampo. Abuso sexual y factores asociados en población escolarizada perteneciente a una institución educativa de la ciudad de Manizales, Caldas (Colombia), $2012 . \quad$ Lúmina. 2013;13(1):47-60. Https://www.doi.org/10.30554/archmed.13.1.12.2013

17. Rico ED, Paternain N, Portillo N, Galarza AL. Análisis de la relación entre factores interpersonales y riesgo suicida en adolescentes de la ciudad de Necochea. Perspect En Psicol Rev Psicol Cienc Afines. 2016;13(2):95-106. URL. 